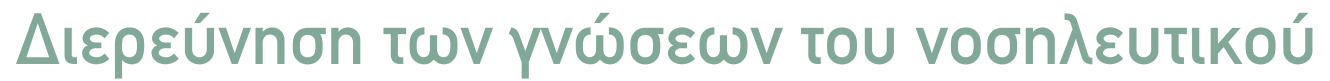

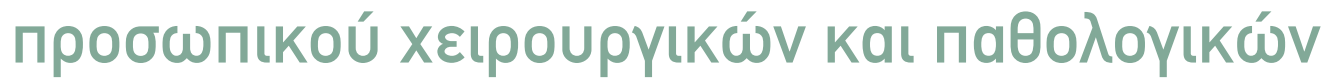

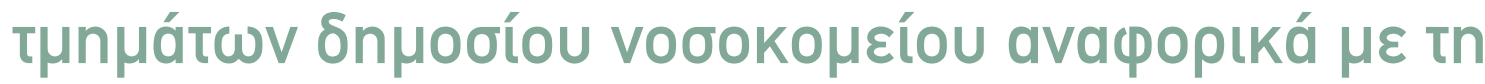

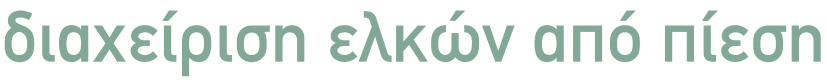

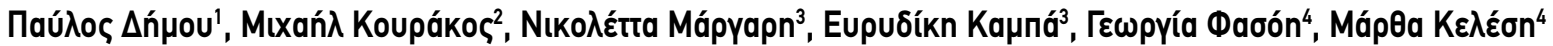

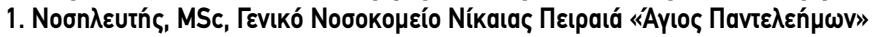

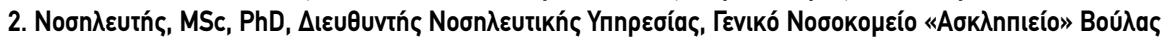

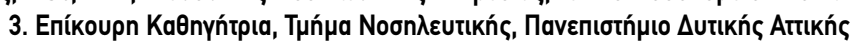

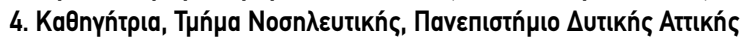

\section{ПEPIAHWH}

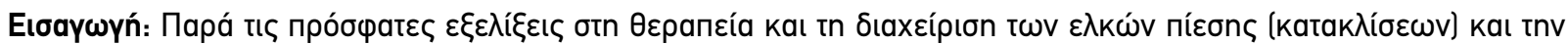

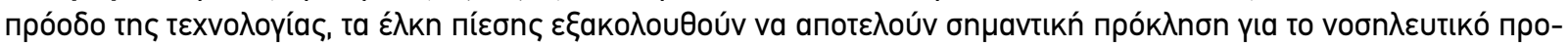

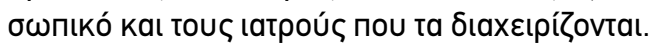

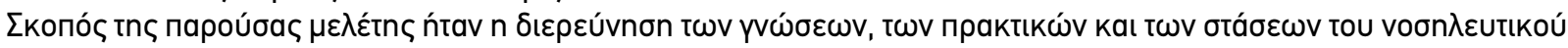

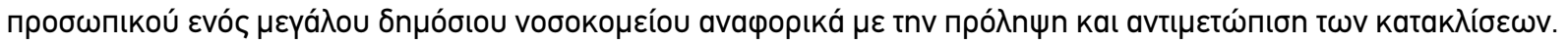

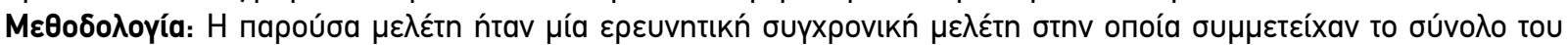

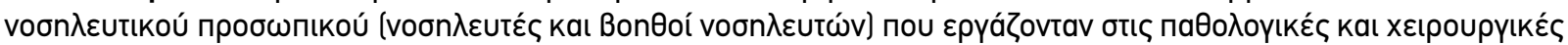

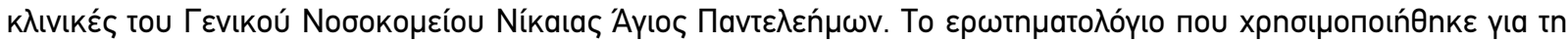

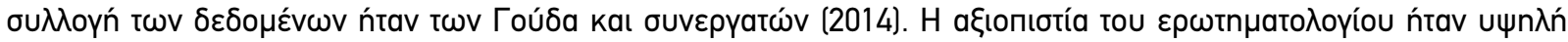

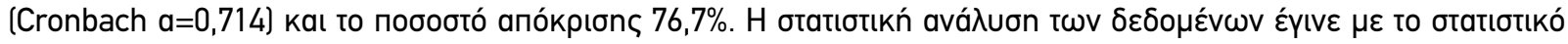

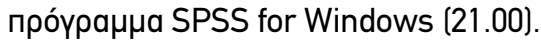

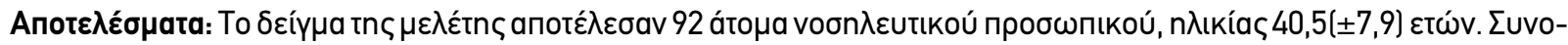

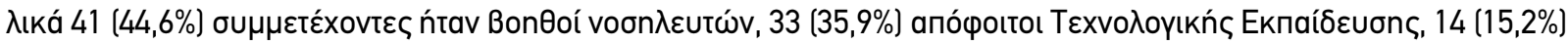

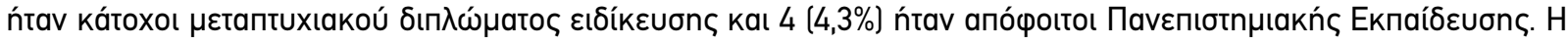

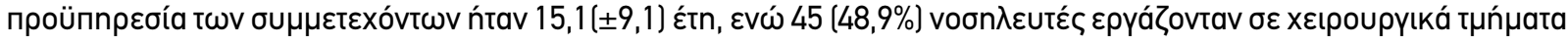

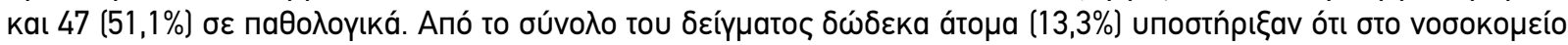

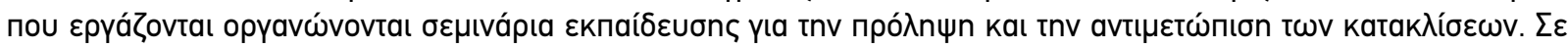

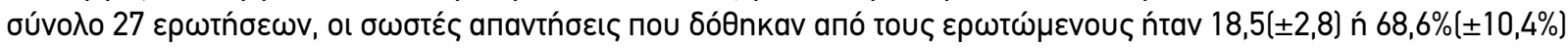

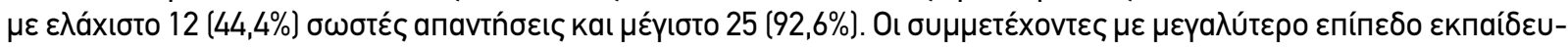

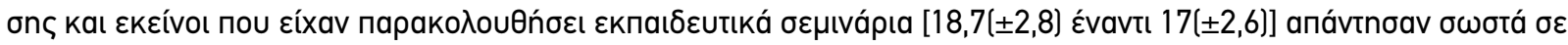

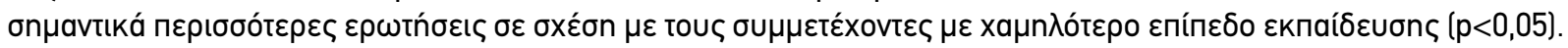

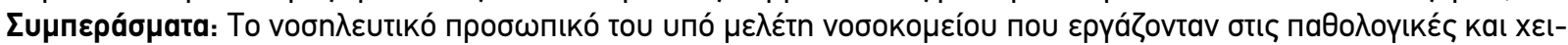

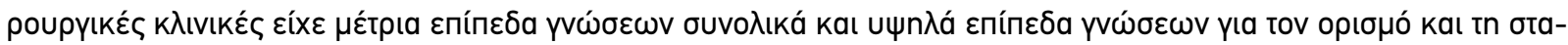

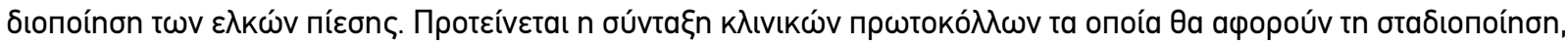

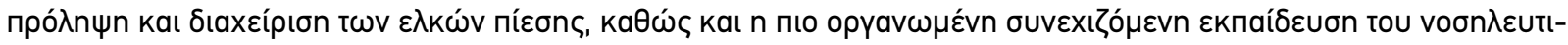
коú пробшпเкоú.

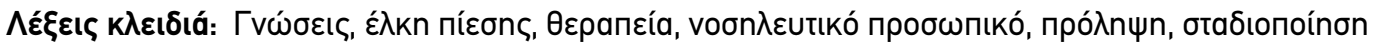




\section{BAEIKA EHMEIA}

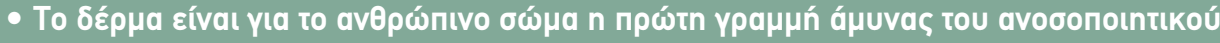

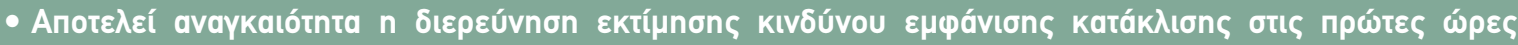

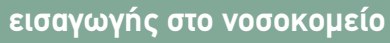

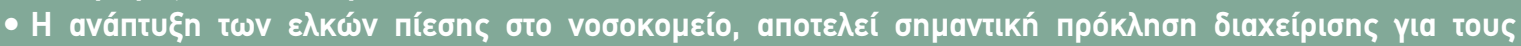

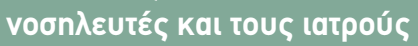

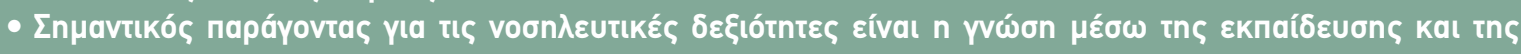
врпвıрі́as

\section{EIIAГRГH}

T

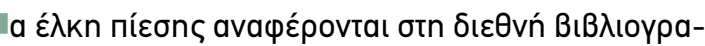

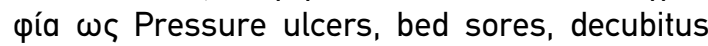
ulcers ń pressure sores (Wake 2010) kaı tpau-

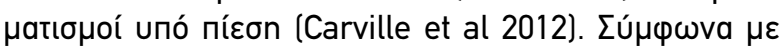

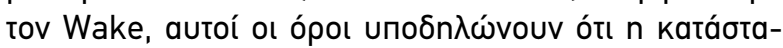

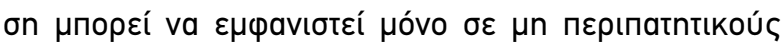

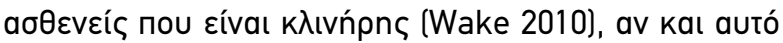

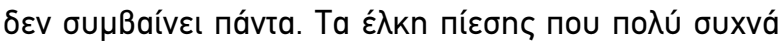

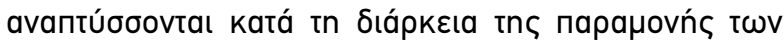

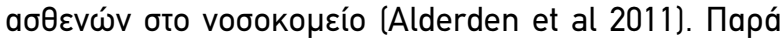

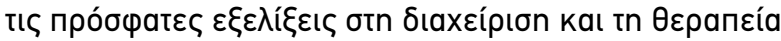

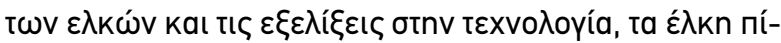

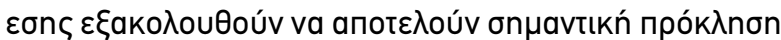

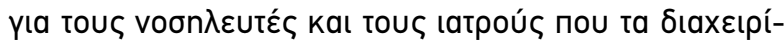

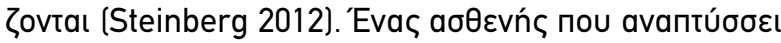

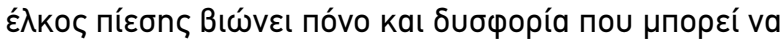

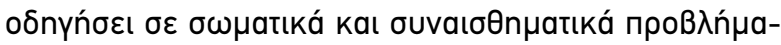

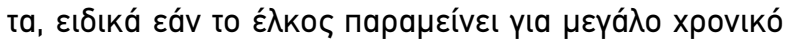

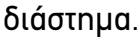

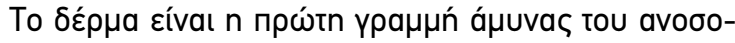

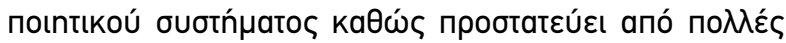

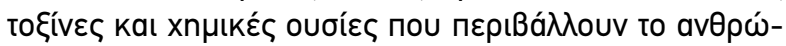

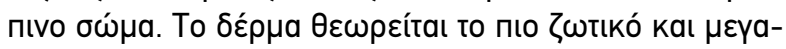

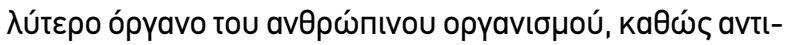

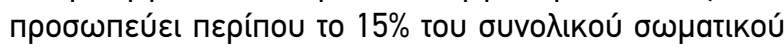

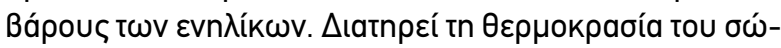

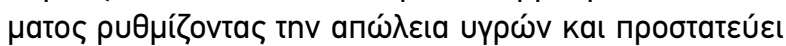

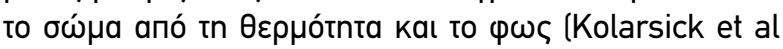

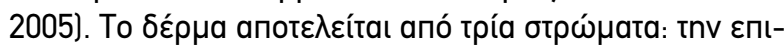

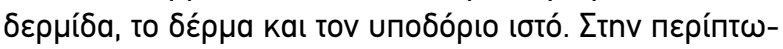

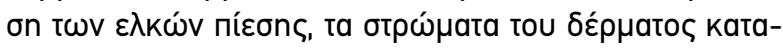

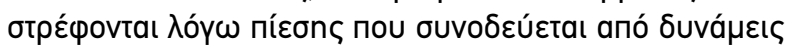

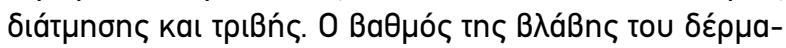

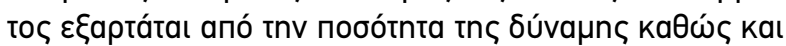

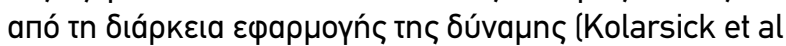
2005).

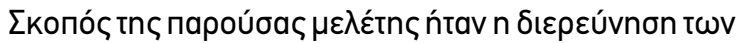

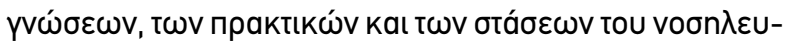

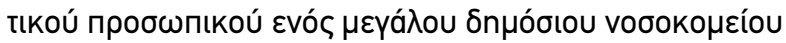

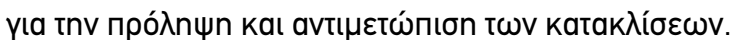

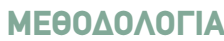

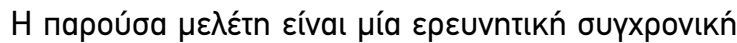

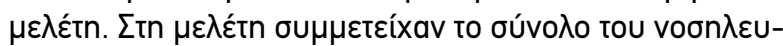

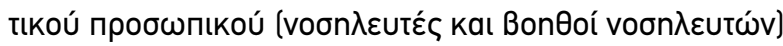

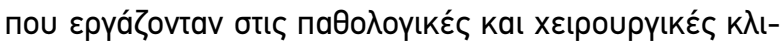

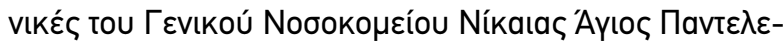
$n \omega \omega v$.

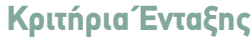

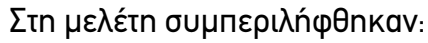

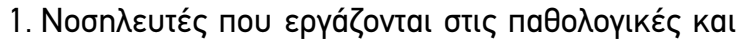

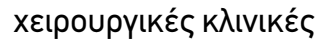

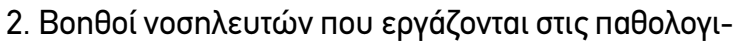

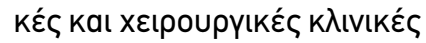

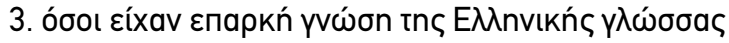

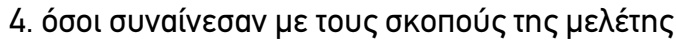

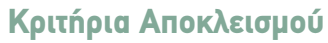

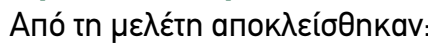

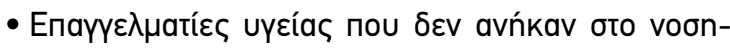

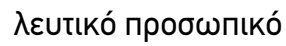

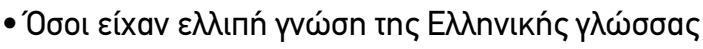

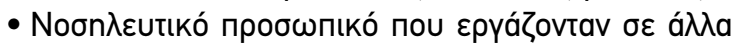

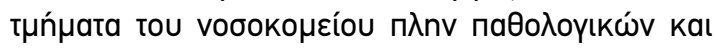

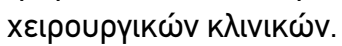

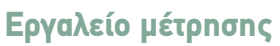

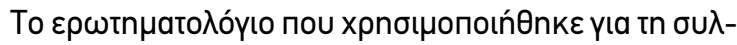

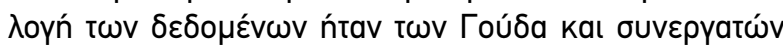

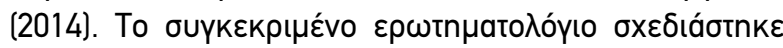

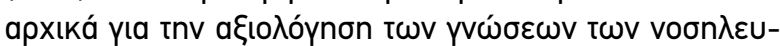

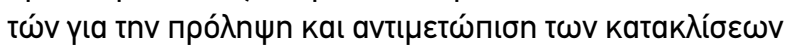

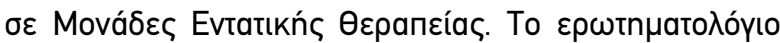

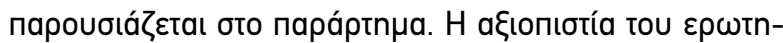

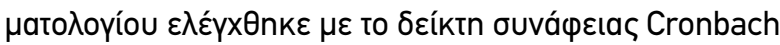

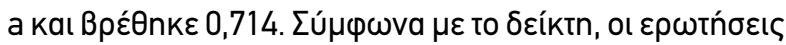

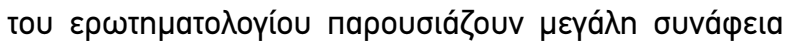
$\mu \varepsilon t a \xi u ́$ touc (van Saane et al 2003). 


\section{EPEYNHTIKH EPГAEIA}

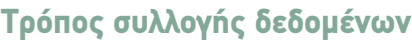

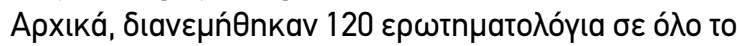

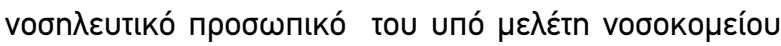

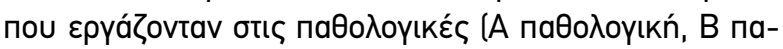

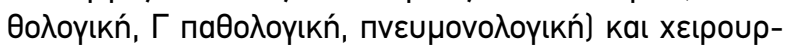

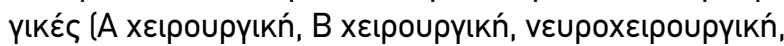

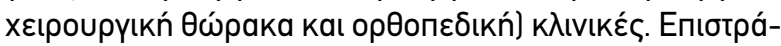

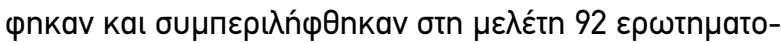

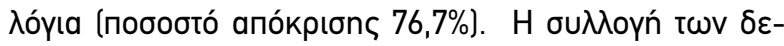

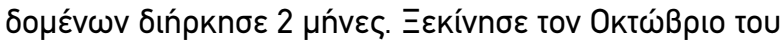

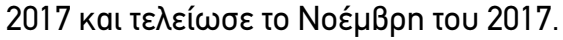

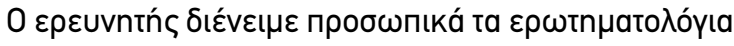

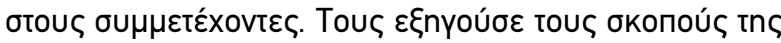

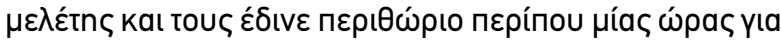

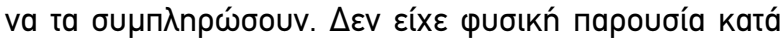

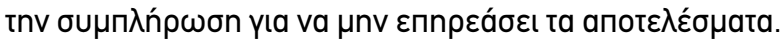

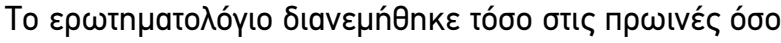

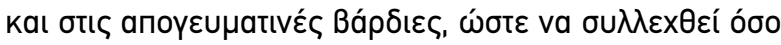

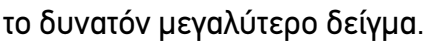

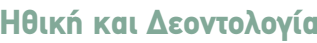

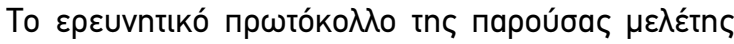

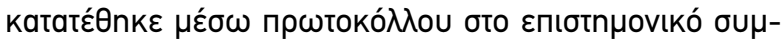

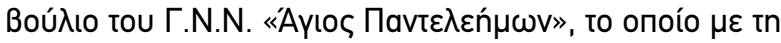

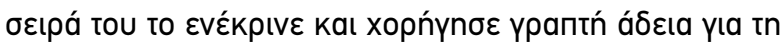

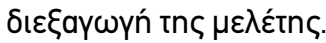

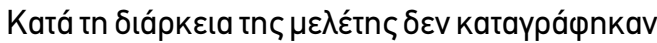

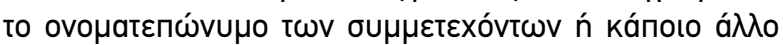

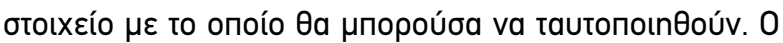

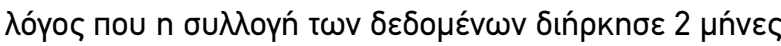

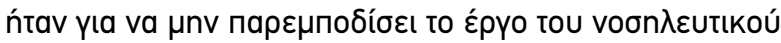

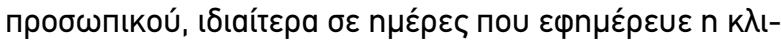

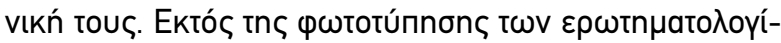

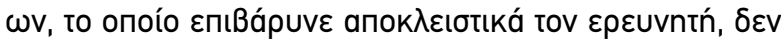

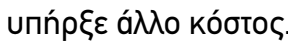

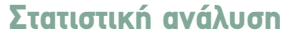

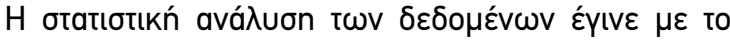

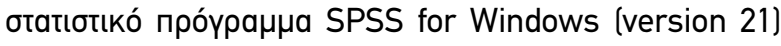
statistical software (SPSS Inc., Chicago, IL). Арxıкá uпо-

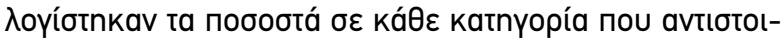

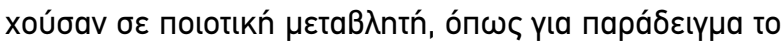

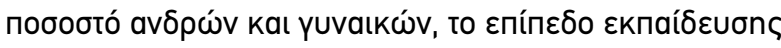

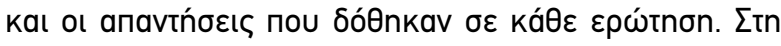

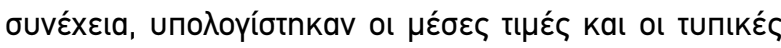

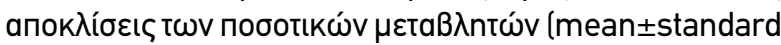

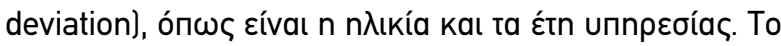

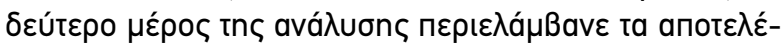

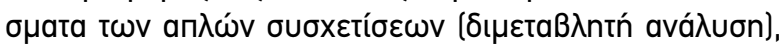

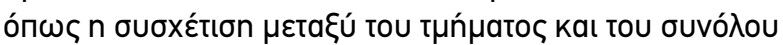

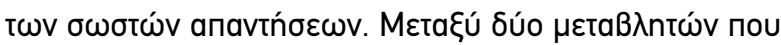

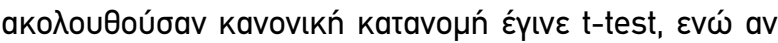

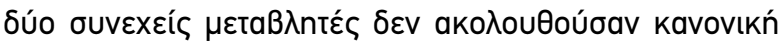

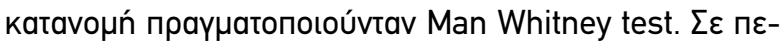

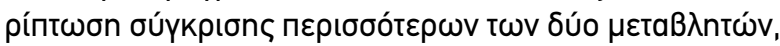

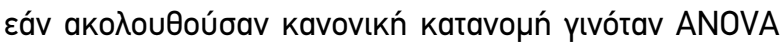

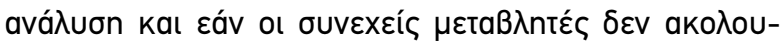

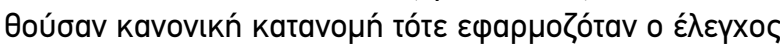

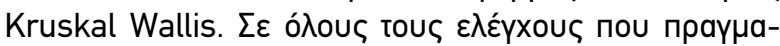

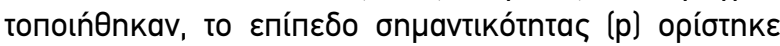

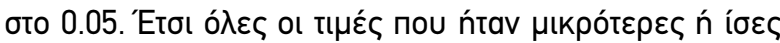

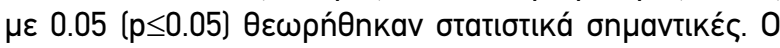

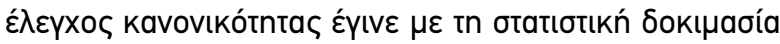
Kolmogorov-Smirnov.

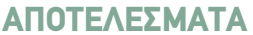

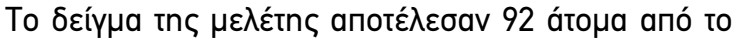

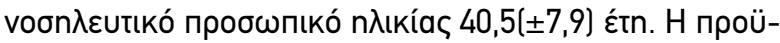

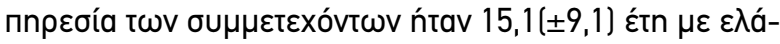

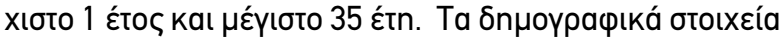

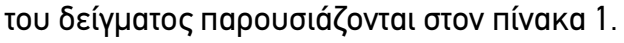

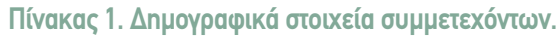

\begin{tabular}{|c|c|c|}
\hline \multicolumn{2}{|l|}{ Metaß入ntи́ } & \multirow{2}{*}{$\begin{array}{l}\mathbf{N}(\%) \\
9(9,8 \%)\end{array}$} \\
\hline Фúlo & 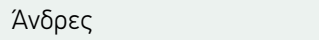 & \\
\hline & Гuvaíkعৎ & $83(90,2 \%)$ \\
\hline \multirow{6}{*}{ 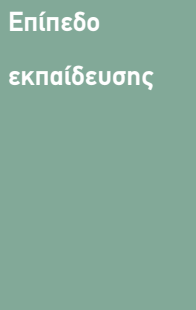 } & BonӨoí voon $\lambda \varepsilon \cup t \omega ́ v$ & $41(44,6 \%)$ \\
\hline & 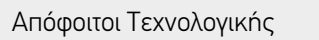 & \\
\hline & 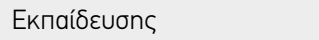 & $33(35,9 \%)$ \\
\hline & 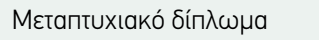 & $14(15,2 \%)$ \\
\hline & 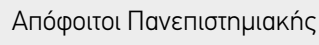 & \\
\hline & 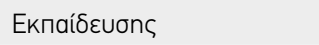 & $4(4,3 \%)$ \\
\hline \multirow[t]{2}{*}{ 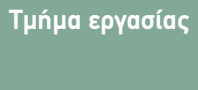 } & Хعıроuрүıкó & $45(48,9 \%)$ \\
\hline & ПаӨо入оүчко́ & $47(51,1 \%)$ \\
\hline
\end{tabular}

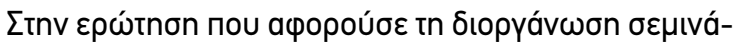

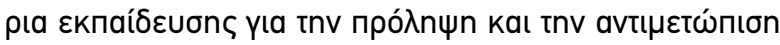

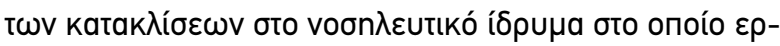

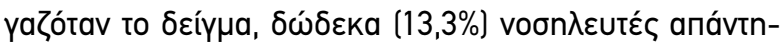

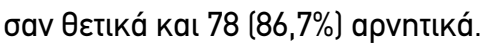

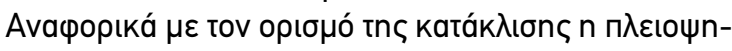

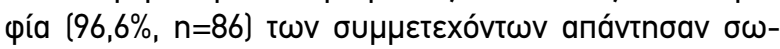

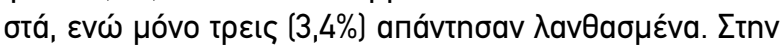

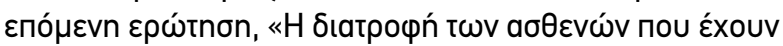

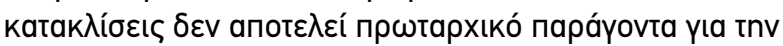

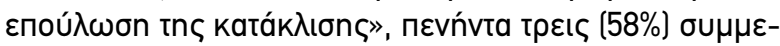

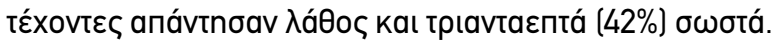

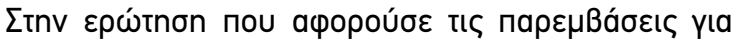

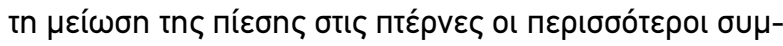

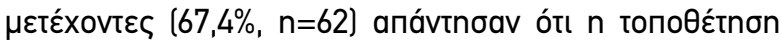

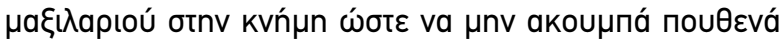

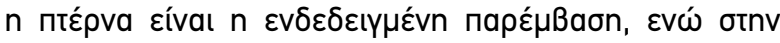

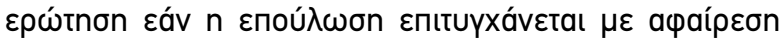




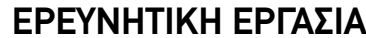

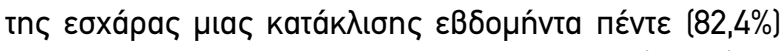

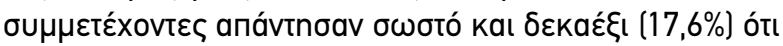

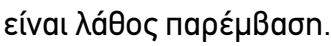

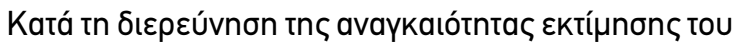

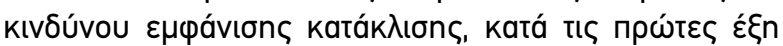

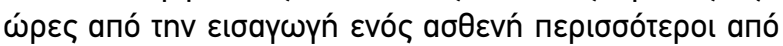

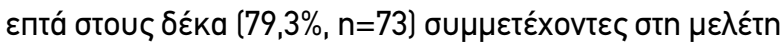

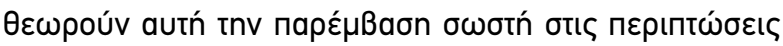

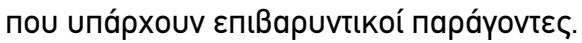

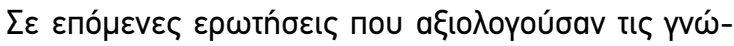

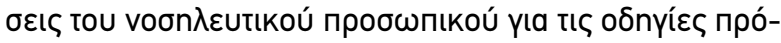

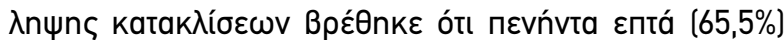

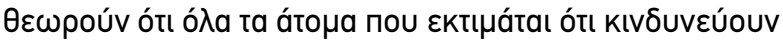

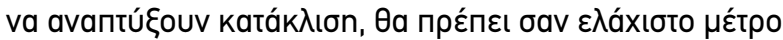

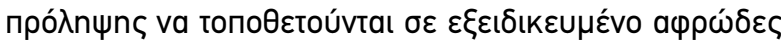

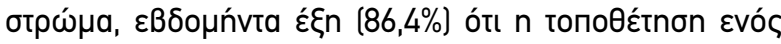

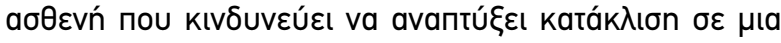

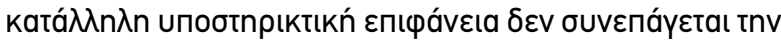

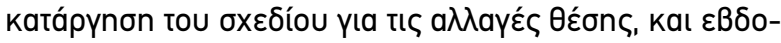

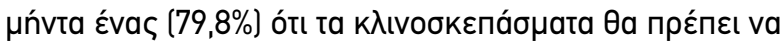

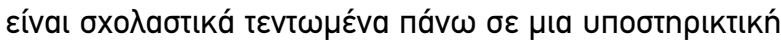

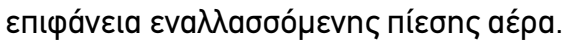

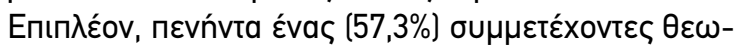

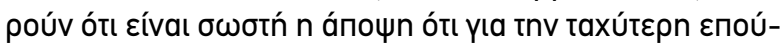

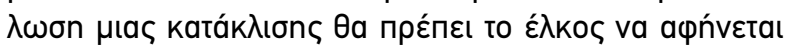

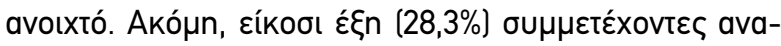

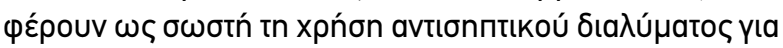

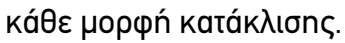

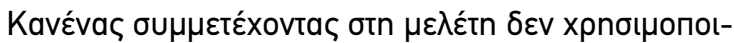

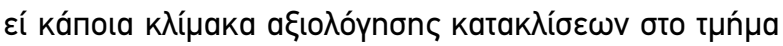

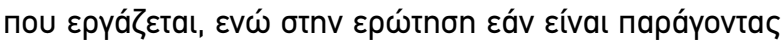

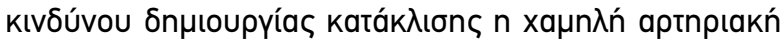

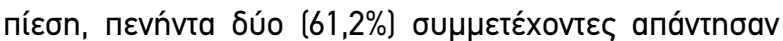

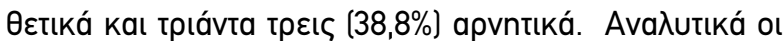

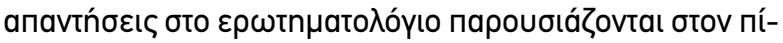
vaka 2.

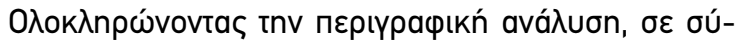

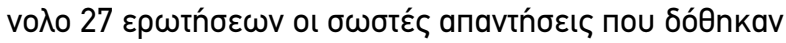

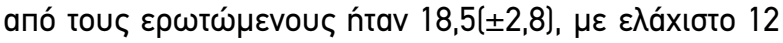

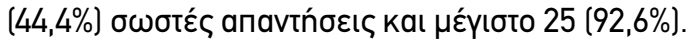

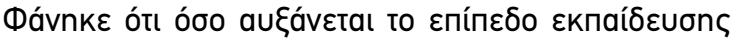

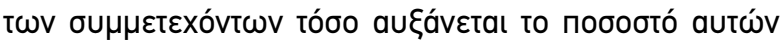

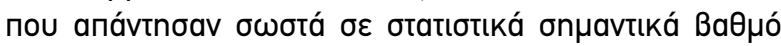

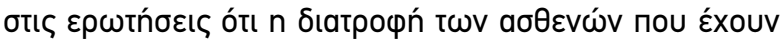

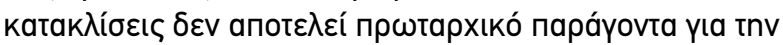

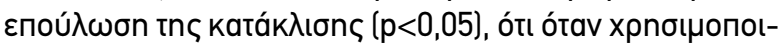

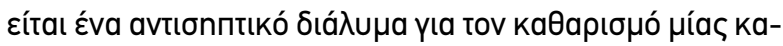

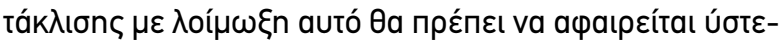

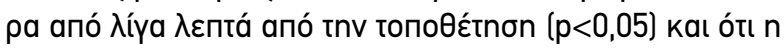

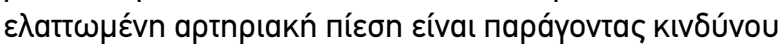

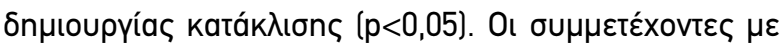

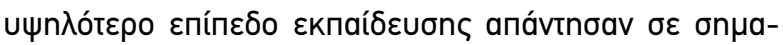

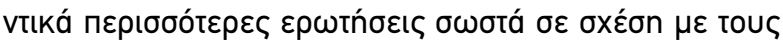

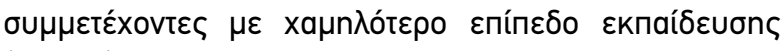

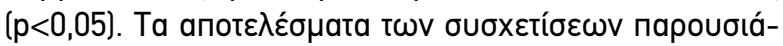
Zovtat otov пívaka 3.

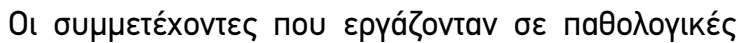

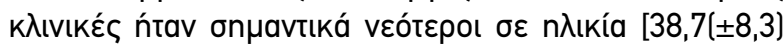

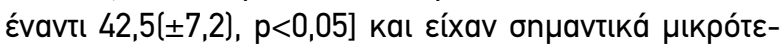

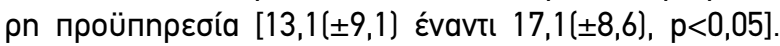

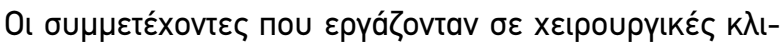

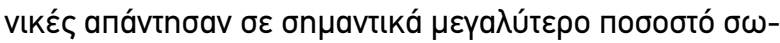

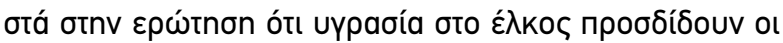

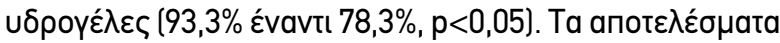

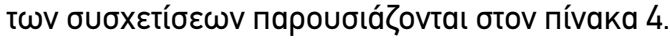

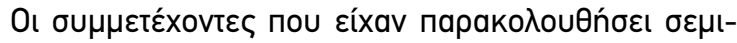

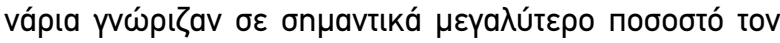

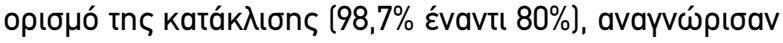

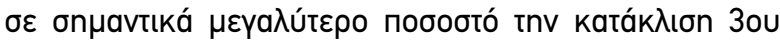

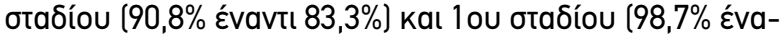

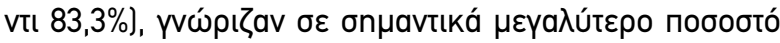

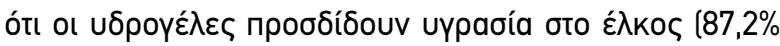

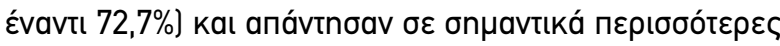

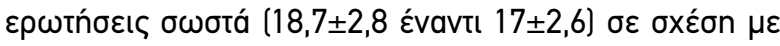

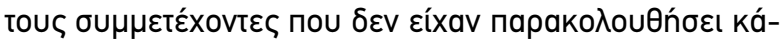

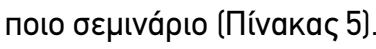

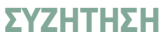

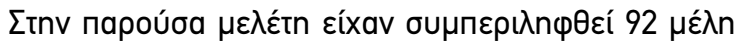

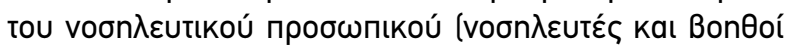

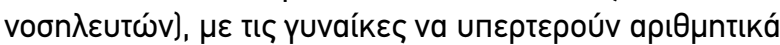

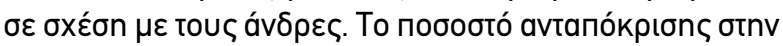

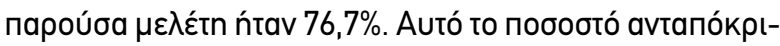

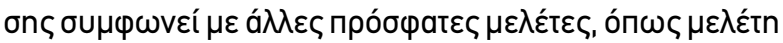

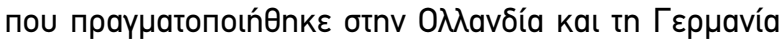

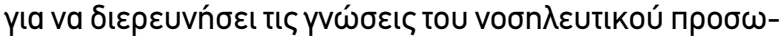

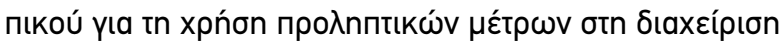

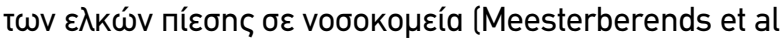

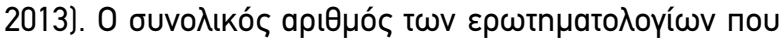

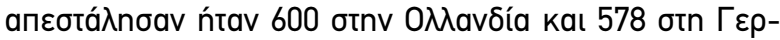

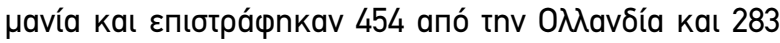

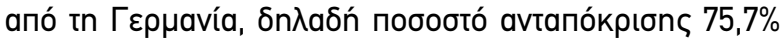
kat $48,4 \%$ aví́otouxa (Meesterberends et al 2013). $\Sigma \varepsilon$

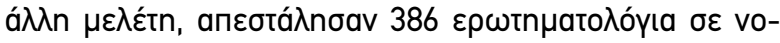

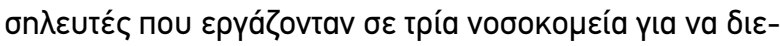

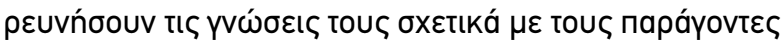

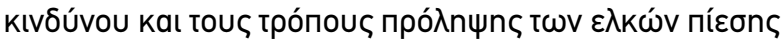
(Tubaishat \& Aljezawi 2014). Enıбрápnkav $263 \varepsilon \rho \omega t n-$

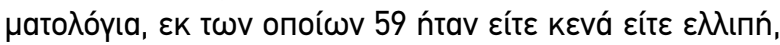

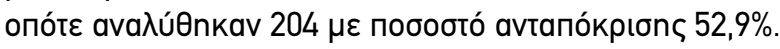

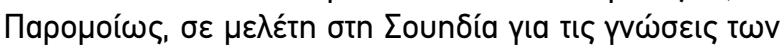

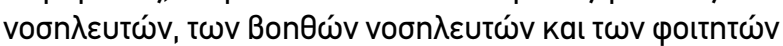

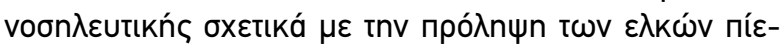

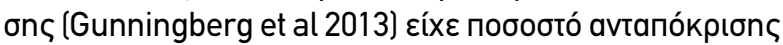

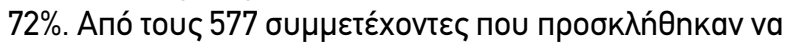

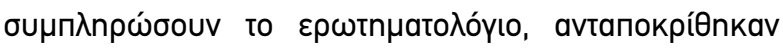




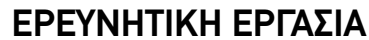

418.

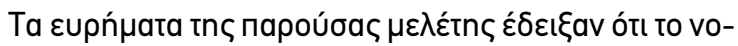

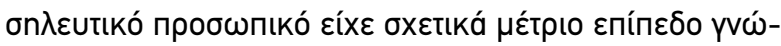

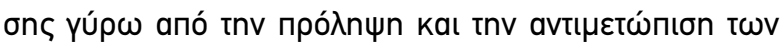

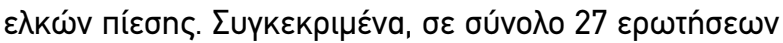

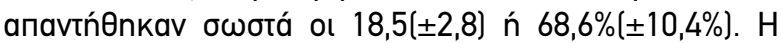

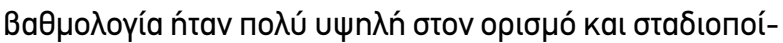

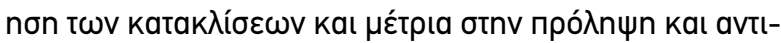

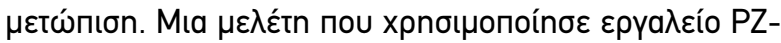

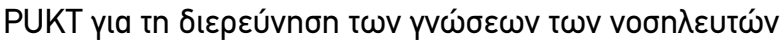

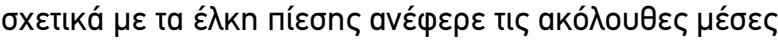

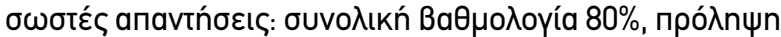
$77 \%$, otáon $86 \%$ kat tpaúpata $77 \%$ (Pieper \& Zulkowski 2014).

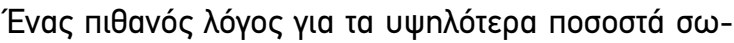

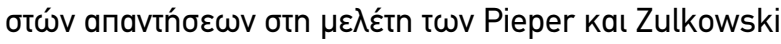

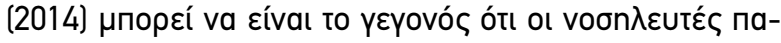

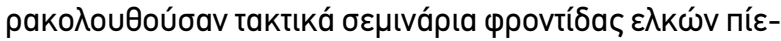

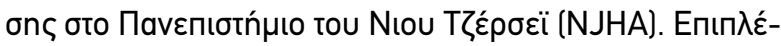

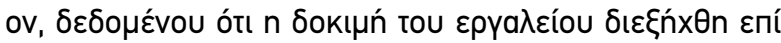

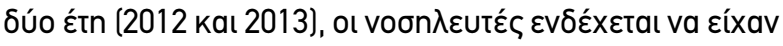

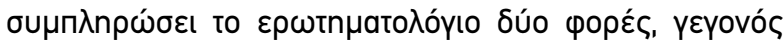

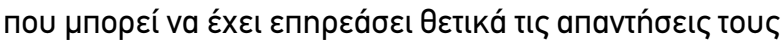

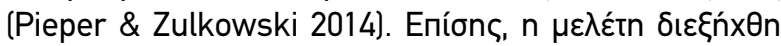

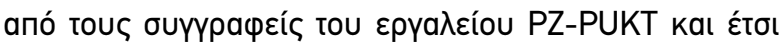

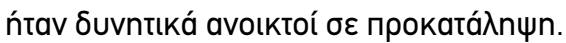

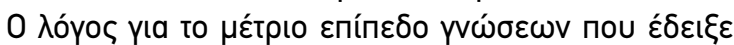

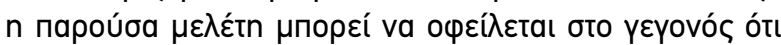

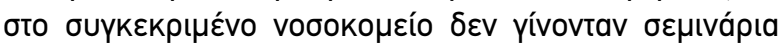

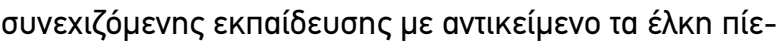

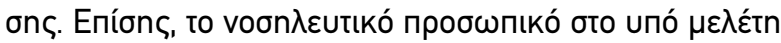

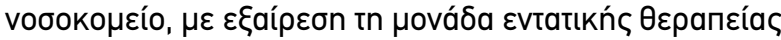

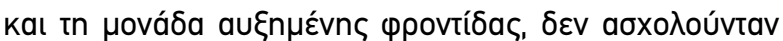

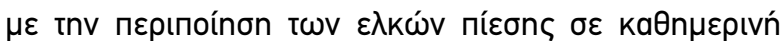

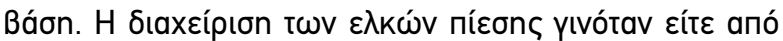

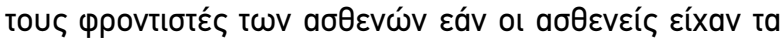

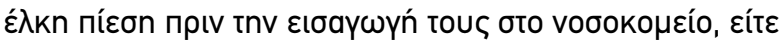
anó touৎ ıatpoú

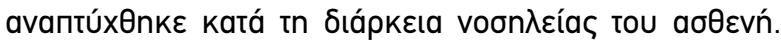

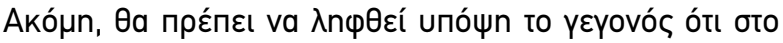

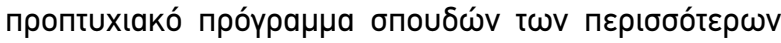

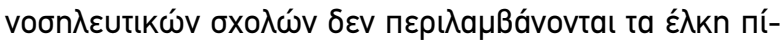

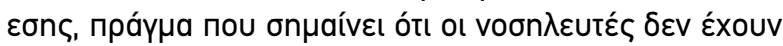

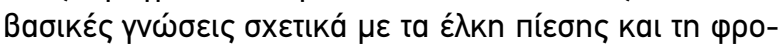

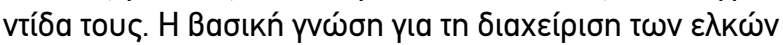

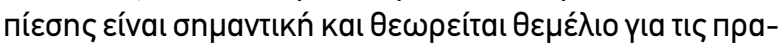

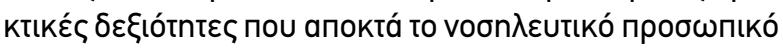

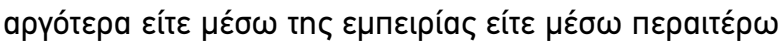
katáptıons. H íoıa apxń toví̧દtaı anó tov Bloom (1956)

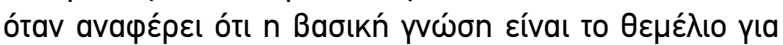

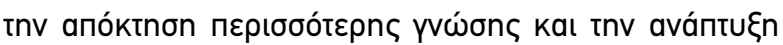

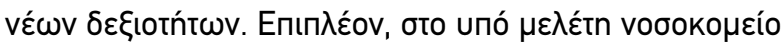

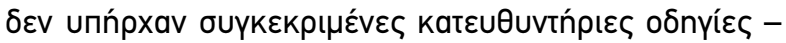

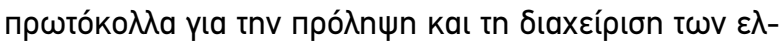

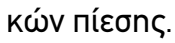

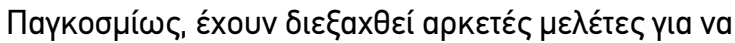

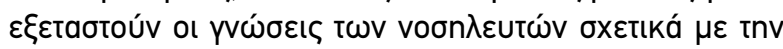

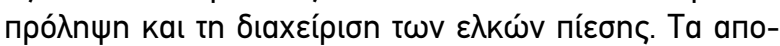

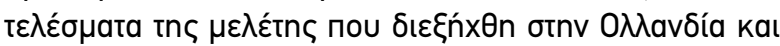

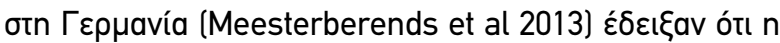

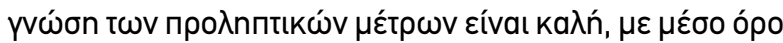

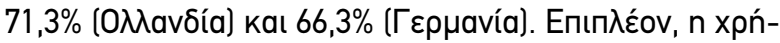

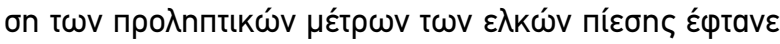

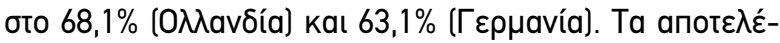

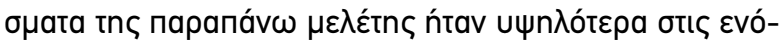

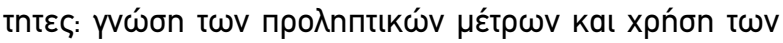

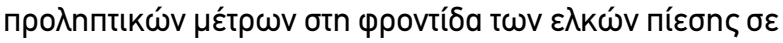

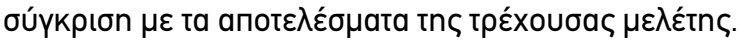

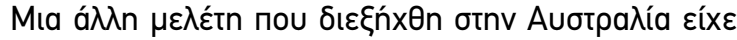

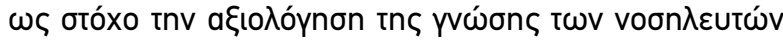

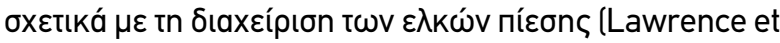

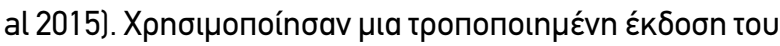

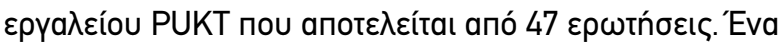

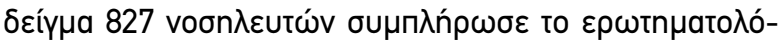

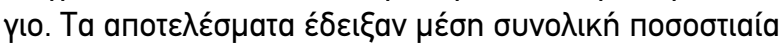

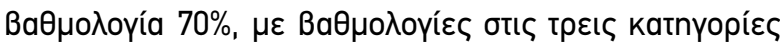

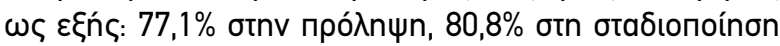

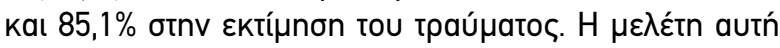

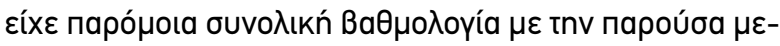
$\lambda \varepsilon ́$ tn $(70 \%$ Évavtı 68,6\%).

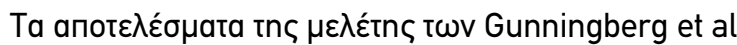

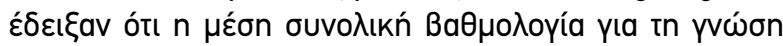

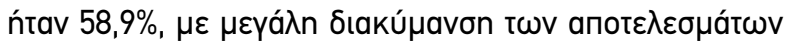

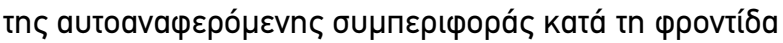

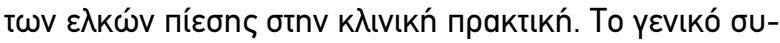

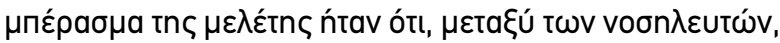

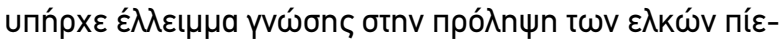
ons (Gunningberg et al 2013).

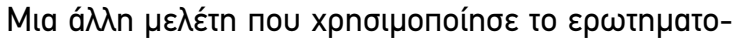

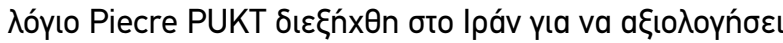

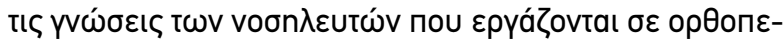

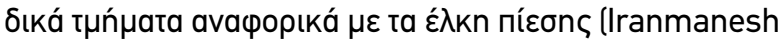

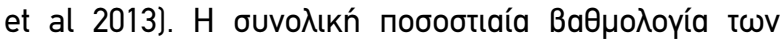

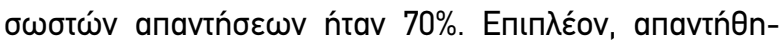

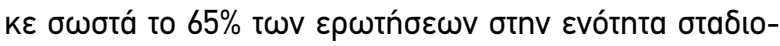

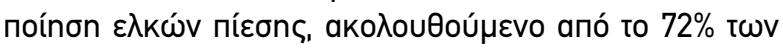

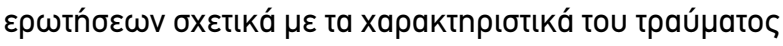

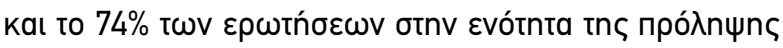

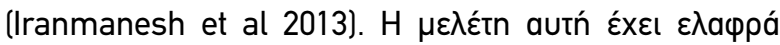

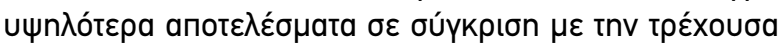

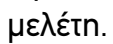

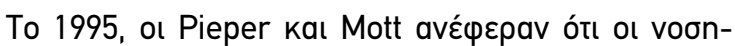

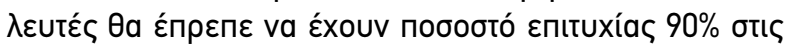

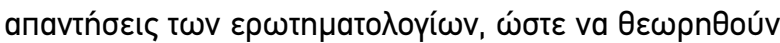

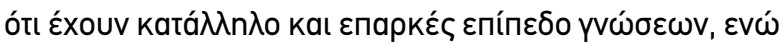

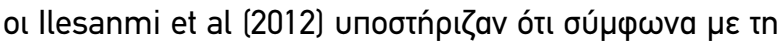




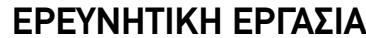

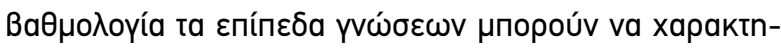

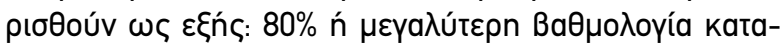

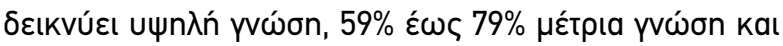

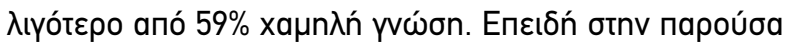

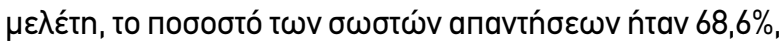

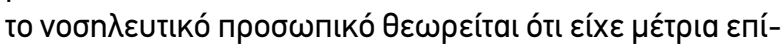

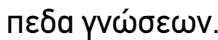

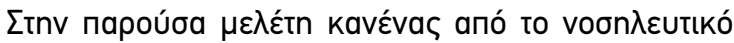

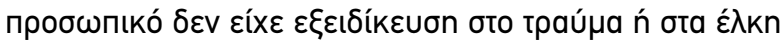

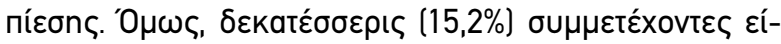

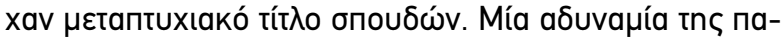

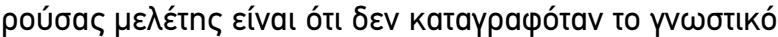

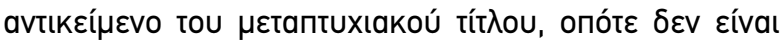

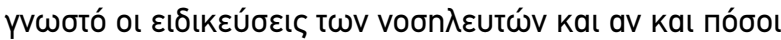

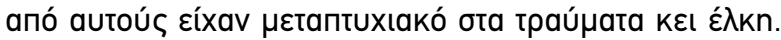

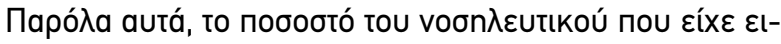

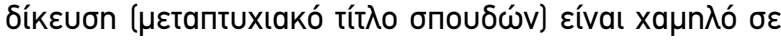

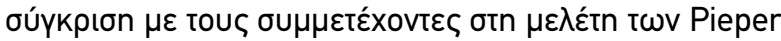

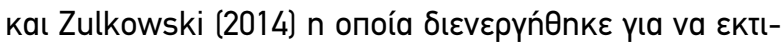

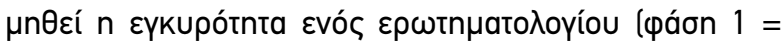
$61 \%$ kaı páon 2 = 48\%).

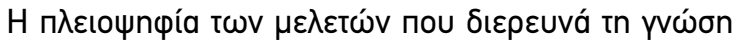

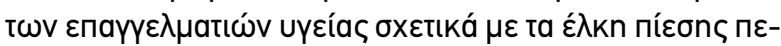

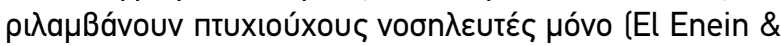
Zaghloul, 2011, Tubaishat \& Aljezawi, 2014, Lawrence et

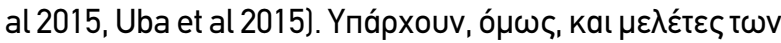

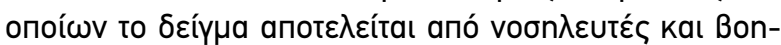

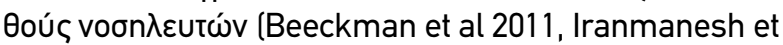

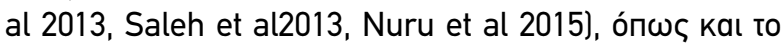

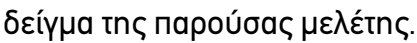

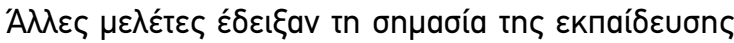

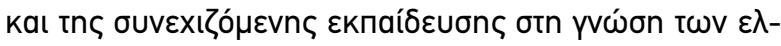

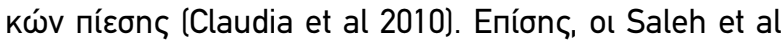

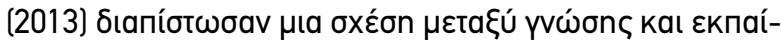

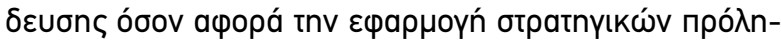

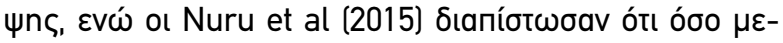

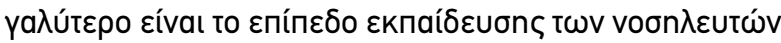

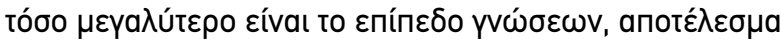

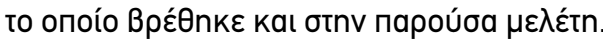

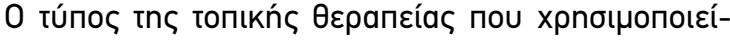

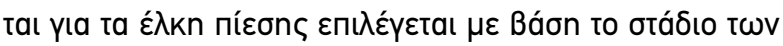

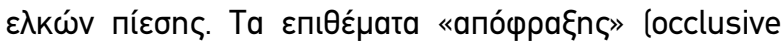

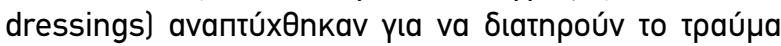

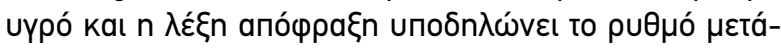

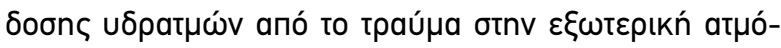

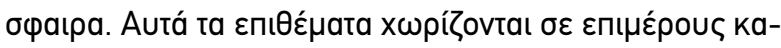

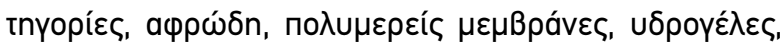

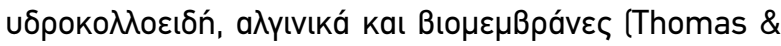

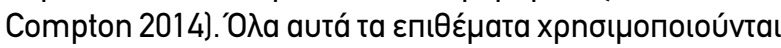

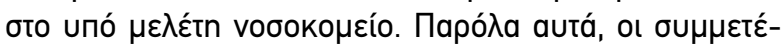

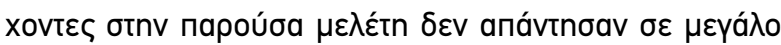

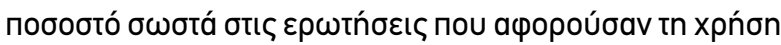

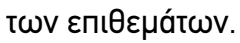

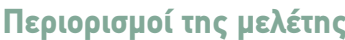

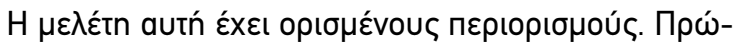

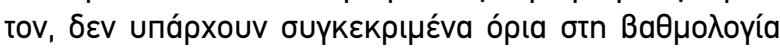

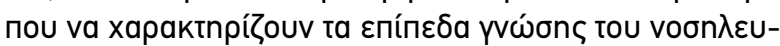

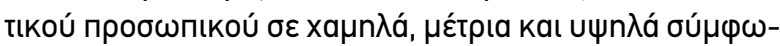

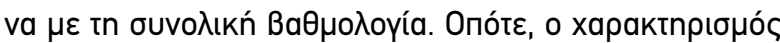

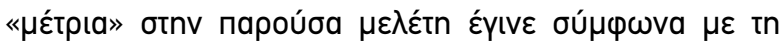

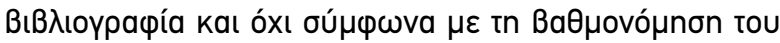

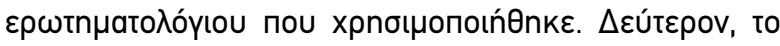

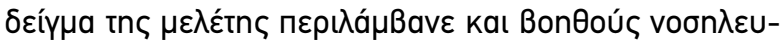

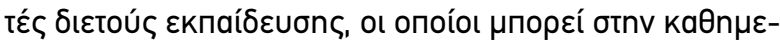

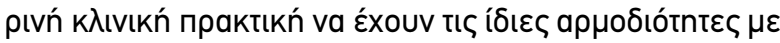

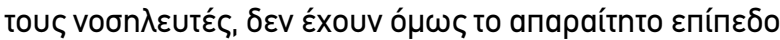
Ө

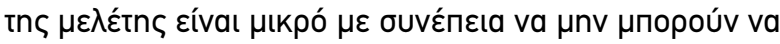

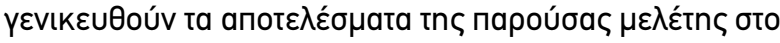

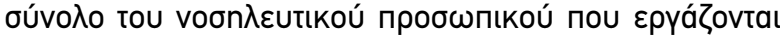

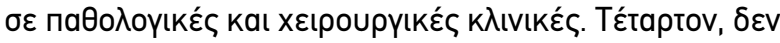

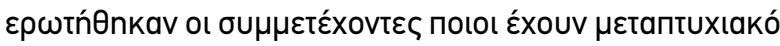

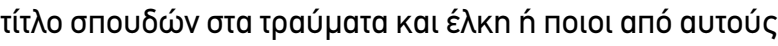

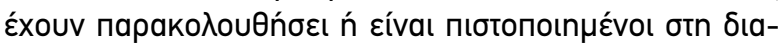

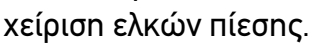

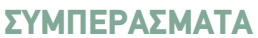

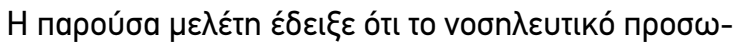

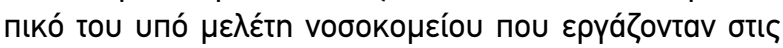

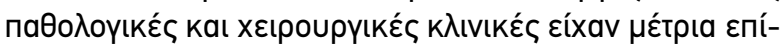

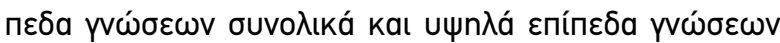

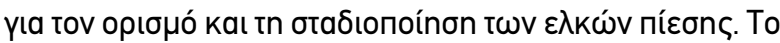

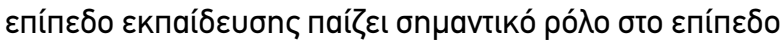

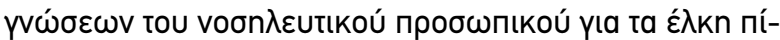

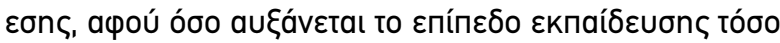

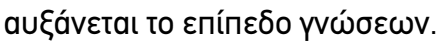

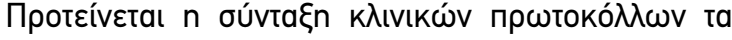

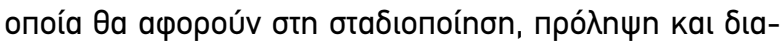

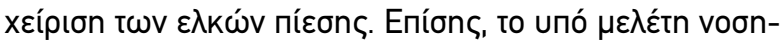

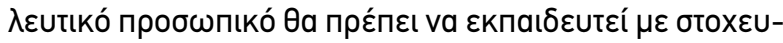

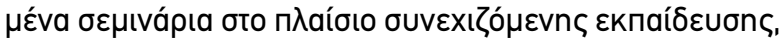

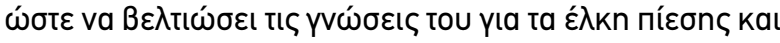

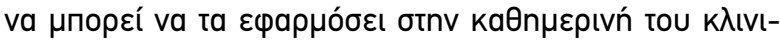

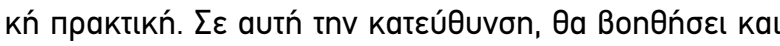

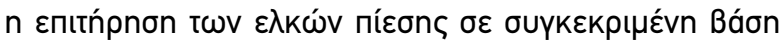

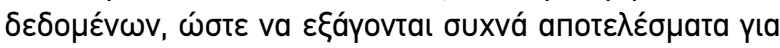

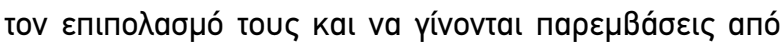

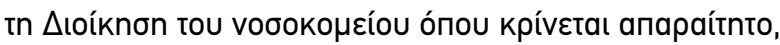

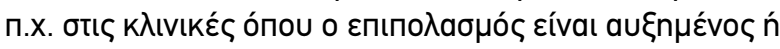

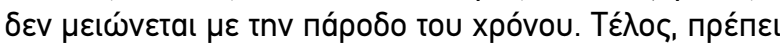

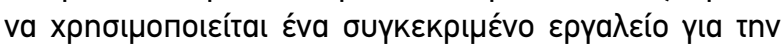

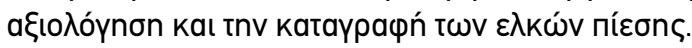

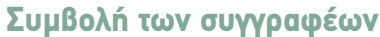

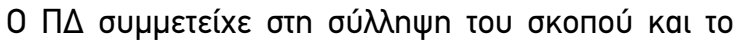




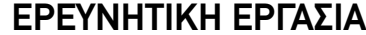

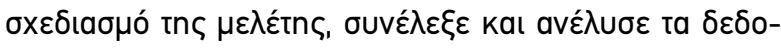

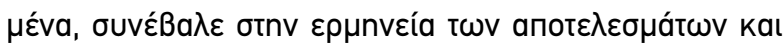

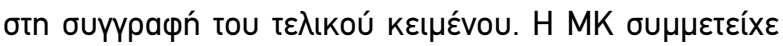

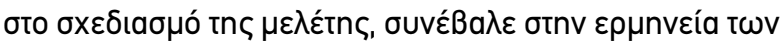

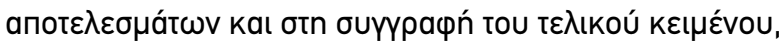

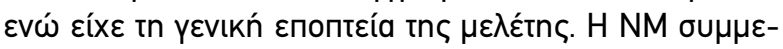

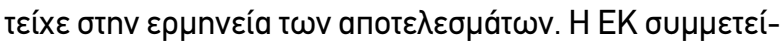

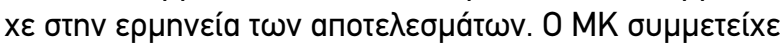

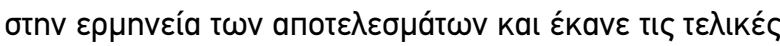

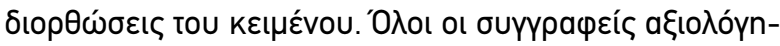

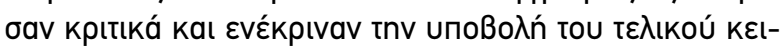
$\mu \varepsilon ́$ vou.

\section{ВІВАІОГРАФІА}

Alderden J., Whitney J.D., Taylor S.M. \& Zaratkiewicz S. (2011). Risk profile characteristics associated with outcomes of hospitalacquired pressure ulcers: a retrospective review. Critical care nurse 31:30-43.

Beeckman D., Defloor T., Schoonhoven L. \& Vanderwee K. (2011). Knowledge and Attitudes of Nurses on Pressure Ulcer Prevention: A Cross-Sectional Multicenter Study in Belgian Hospitals. Worldviews on Evidence-Based Nursing 8:166-176.

Bloom B.S. (1956). Taxonomy of educational objectives: The classification of educational goals. Handbook I: Cognitive domain., New York: David McKay Company.

Carville K. (2012). Pan Pacific Clinical Practice Guideline for the Prevention and Management of Pressure Injury, Australia.

Claudia G., Diane M., Daphney S.G. \& Danièle D. (2010). Prevention and treatment of pressure ulcers in a university hospital centre: a correlational study examining nurses' knowledge and best practice. International journal of nursing practice 16:183-187.

El Enein N.Y.A. \& Zaghloul A.A. (2011). Nurses' knowledge of prevention and management of pressure ulcer at a Health Insurance Hospital in Alexandria. International Journal of Nursing Practice 17: 262-268.

Gunningberg L., Mårtensson G., Mamhidir A.G., Florin J., Muntlin Athlin A. \& Bååth C. (2013). Pressure ulcer knowledge of registered nurses, assistant nurses and student nurses: A descriptive, comparative multicentre study in Sweden. International Wound Journal 1:1-7.

Ilesanmi R., Ofi B. \& Adejumo P. (2012). Nurses' knowledge of pressure ulcer prevention in Ogun State, Nigeria: results of pilot study. Ostomy Wound Management 58:24-32.

Iranmanesh S., Tafti A.A., Rafiei H., Dehghan M. \& Razban F. (2013). Orthopaedic nurses' knowledge about pressure ulcers in Iran: a cross-sectional study. Journal of Wound Care 22:1-5.

Kolarsick P.A.J., Kolarsick M.A. \& Goodwin C. (2005). Anatomy and physiology of the skin. Dermatology nursing / Dermatology Nurses' Association 17:62.

Lawrence P., Fulbrook P. \& Miles S. (2015). A Survey of Australian
Nurses' Knowledge of Pressure Injury/Pressure Ulcer Management. Journal of Wound, Ostomy and Continence Nursing Society 2:1-11.

Meesterberends E., Wilborn D., Lohrmann C., Schols J.M. \& Halfens R.J. (2013). Knowledge and use of pressure ulcer preventive measures in nursing homes: A comparison of Dutch and German nursing staff. Journal of Clinical Nursing 1:1-11.

Nuru N., Zewdu F., Amsalu S. \& Mehretie Y. (2015). Knowledge and practice of nurses towards prevention of pressure ulcer and associated factors in Gondar University Hospital, Northwest Ethiopia. BMC nursing 14:1-8.

Pieper B. \& Zulkowski K. (2014). The pieper-zulkowski pressure ulcer knowledge test. Advances in skin \& wound care 27:413-20.

Saleh M.Y.N., Al-Hussami M. \& Anthony D. (2013). Pressure ulcer prevention and treatment knowledge of Jordanian nurses. Journal of tissue viability 22:1-11.

Steinberg K. (2012). Pressure ulcers: An ongoing problem. Care Management (Summer) 1:10-12.

Thomas D. \& Compton G. (2014). Pressure Ulcers in the Aging Population, Available at: http://link.springer.com/10.1007/978-162703-700-6\nhttp://link.springer.com/content/pdf/10.1007/9781-62703-700-6.pdf.

Tubaishat A. \& Aljezawi M. (2014). Exploring Pressure Ulcer Care in Jordan: Nurses' Knowledge and Practice. Journal of the Dermatology Nurses' Association 6:115-123.

Uba M., Alih F.I., Kever R.T. \& Lola N. (2015). Knowledge, attitude and practice of nurses toward pressure ulcer prevention in University of Maiduguri Teaching Hospital, Borno State, North-Eastern, Nigeria. International Journal of Nursing and Midwifery 7:54-60.

van Saane N., Sluiter J.K., Verbeek J.H.A.M. \& Frings-Dresen M.H.W. (2003). Reliability and validity of instruments measuring job satisfaction-a systematic review. Occupational Medicine 53:191200.

Wake W.T. (2010). Pressure ulcers: what clinicians need to know. The Permanente Journal 14:56-60 


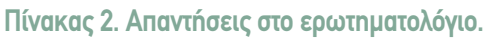

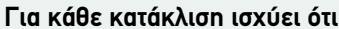

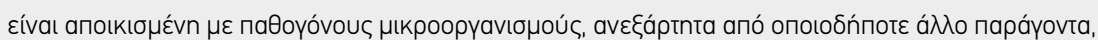

$42 \quad 46,7 \%$

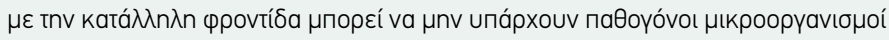

$37 \quad 41,1 \%$

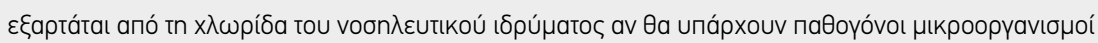

$7 \quad 7,38 \%$

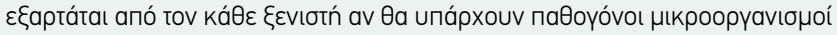

$4 \quad 4,4 \%$

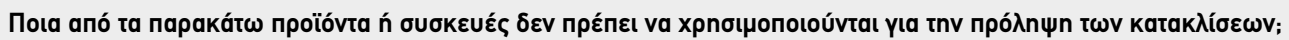

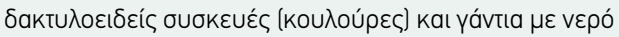

$59 \quad 64,8 \%$

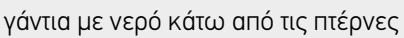

$13 \quad 14,3 \%$

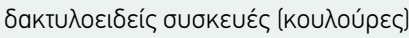

$\begin{array}{ll}7 & 7,7 \%\end{array}$

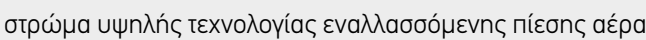

$6 \quad 6,6 \%$

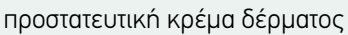

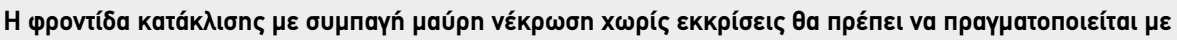

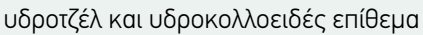

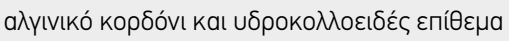

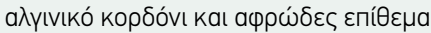

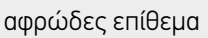

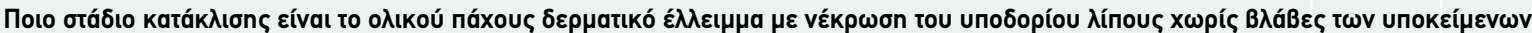

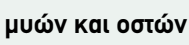

\begin{tabular}{|c|c|}
\hline 10u otaסíou & 2 \\
\hline 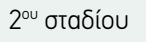 & 1 \\
\hline 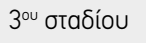 & 81 \\
\hline 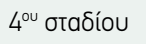 & 6 \\
\hline
\end{tabular}

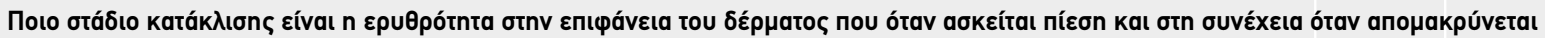

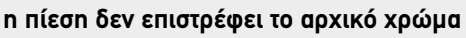

jou otadíou

$87 \quad 96,7 \%$

2ou otadíou

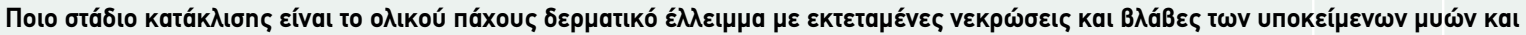
ootẃv

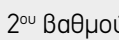

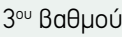

$6 \quad 6,7 \%$

$4^{\circ \mathrm{u}} \mathrm{Ba \theta \mu oú}$

81

$6,7 \%$

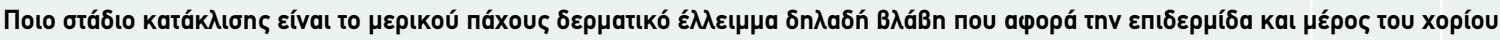

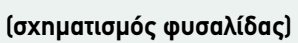

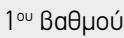

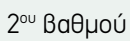

3ou $B a \theta \mu \circ{ }^{\circ}$

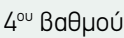

$3 \quad 3,3 \%$

$\begin{array}{ll}3 & 3,3 \%\end{array}$




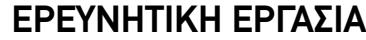

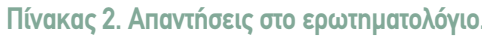

Enuloyéc

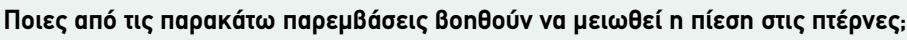

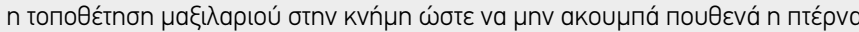

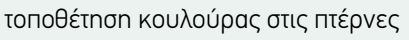

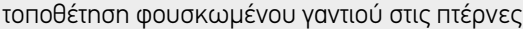

Kavéva anó ta пapanávw

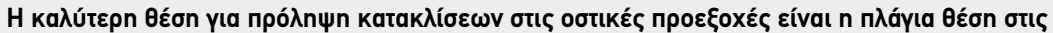

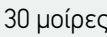

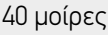

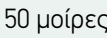

90 ноірєৎ

$41,8 \%$

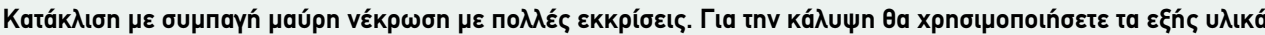

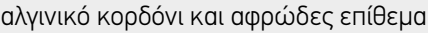

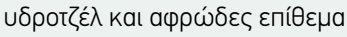

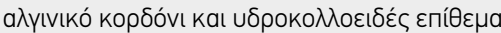

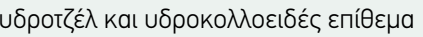

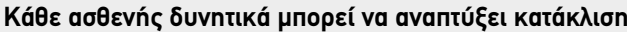

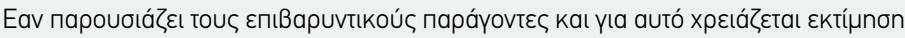

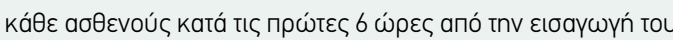

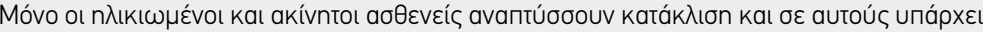

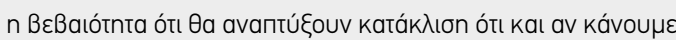

$2,2 \%$

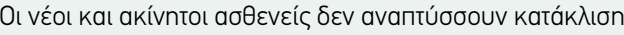

Tínotع aпó ta пapanávw

$2,2 \%$

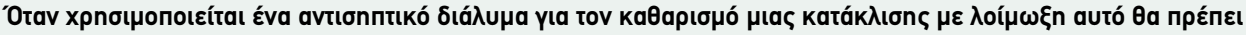

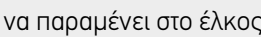

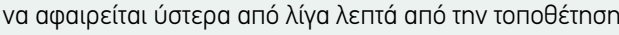

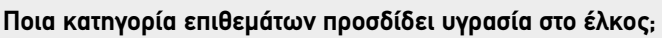

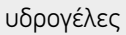

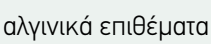

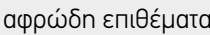

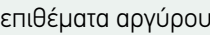

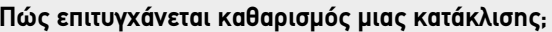

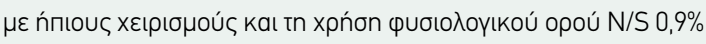

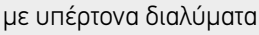

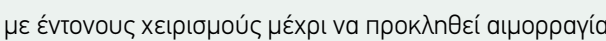

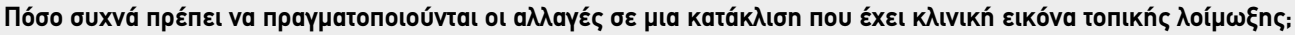

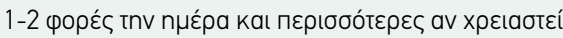

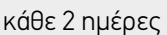

$64 \quad 72,7 \%$

$14 \quad 15,9 \%$

$10 \quad 11,4 \%$

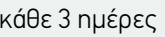

кavéva anó ta пapanávw

$56 \%$

$28,6 \%$

$4,4 \%$

10 


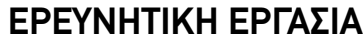

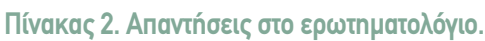

\begin{tabular}{|c|c|c|}
\hline & $\mathbf{N}$ & $\%$ \\
\hline \multicolumn{3}{|l|}{ 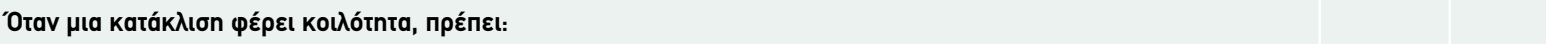 } \\
\hline 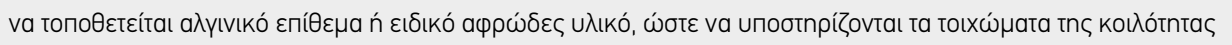 & 54 & $60 \%$ \\
\hline 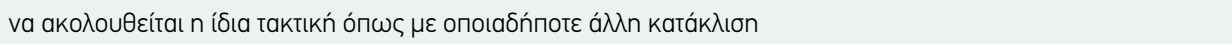 & 10 & $11,1 \%$ \\
\hline 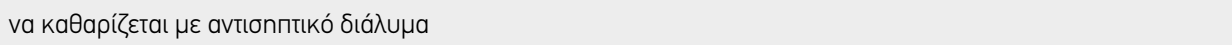 & 1 & $1,1 \%$ \\
\hline 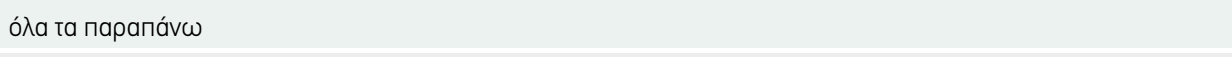 & 25 & $27,8 \%$ \\
\hline \multicolumn{3}{|l|}{ 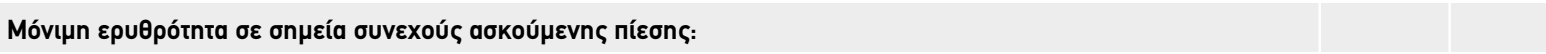 } \\
\hline 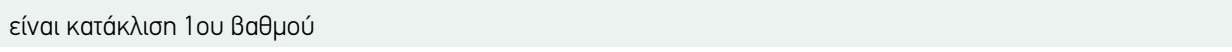 & 86 & $93,5 \%$ \\
\hline 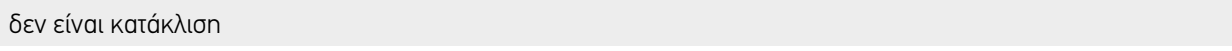 & 6 & $6,5 \%$ \\
\hline
\end{tabular}

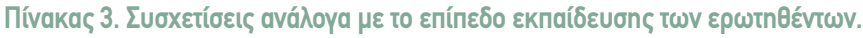

\begin{tabular}{|c|c|c|c|c|c|}
\hline & MET/KO & AEI & ATEI & $\Delta E$ & $\mathbf{P}$ \\
\hline 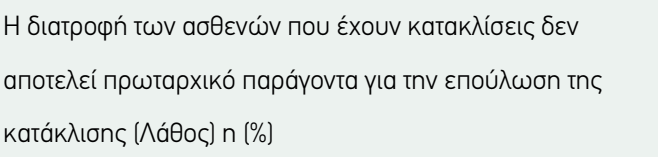 & $\begin{array}{c}12 \\
(85,7 \%)\end{array}$ & $\begin{array}{c}3 \\
(75 \%)\end{array}$ & $\begin{array}{c}19 \\
(61,3 \%)\end{array}$ & $\begin{array}{c}17 \\
(43,6 \%)\end{array}$ & 0,038 \\
\hline 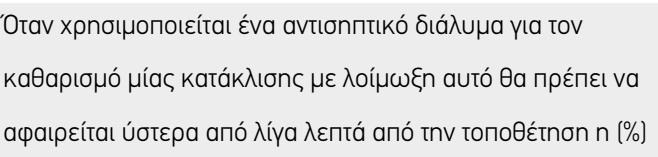 & $\begin{array}{c}14 \\
(100 \%)\end{array}$ & $\begin{array}{c}4 \\
(100 \%)\end{array}$ & $\begin{array}{c}30 \\
(90,9 \%)\end{array}$ & $\begin{array}{c}28 \\
(70 \%)\end{array}$ & 0,049 \\
\hline 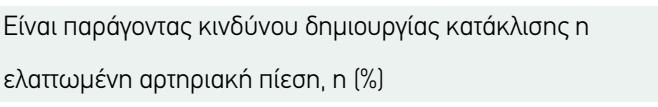 & $\begin{array}{c}12 \\
(85,7 \%)\end{array}$ & $\begin{array}{c}4 \\
(100 \%)\end{array}$ & $\begin{array}{c}14 \\
(48,3 \%)\end{array}$ & $\begin{array}{c}22 \\
(57,9 \%)\end{array}$ & 0,039 \\
\hline 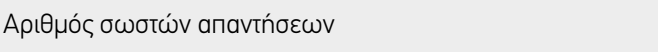 & $20,7 \pm 2,1$ & $19,5 \pm 2,6$ & $18,6 \pm 2,5$ & $17,6 \pm 2,8$ & 0,002 \\
\hline 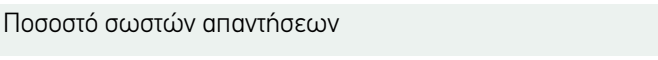 & $76,7 \pm 7,7$ & $72,2 \pm 9,8$ & $69,1 \pm 9,3$ & $65,1 \pm 10,5$ & 0,002 \\
\hline
\end{tabular}




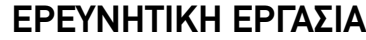

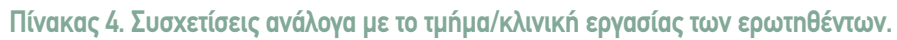

\begin{tabular}{|c|c|c|c|}
\hline & ПаӨоһоуккб́ & Xeıрoupyıкó & $\mathbf{p}$ \\
\hline H入ıkía & $38,7 \pm 8,3$ & $42,5 \pm 7,2$ & 0,022 \\
\hline Проӥпnрєбía & $13,1 \pm 9,1$ & $17,1 \pm 8,6$ & 0,035 \\
\hline 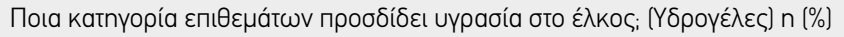 & $36(78,3 \%)$ & $42(93,3 \%)$ & 0,024 \\
\hline
\end{tabular}

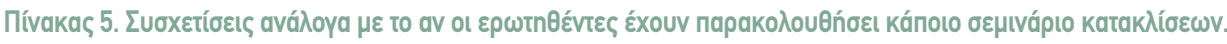

\begin{tabular}{|c|c|c|c|}
\hline & 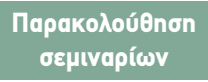 & 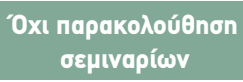 & $\mathbf{p}$ \\
\hline 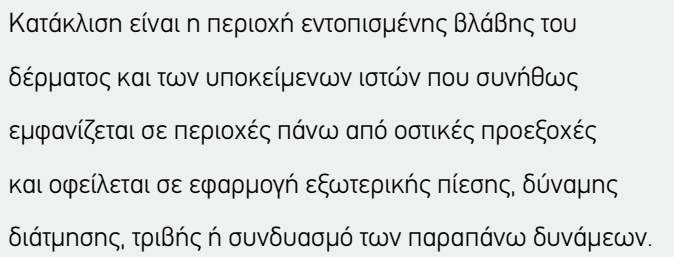 & $76(98,7 \%)$ & $8(80 \%)$ & 0,002 \\
\hline 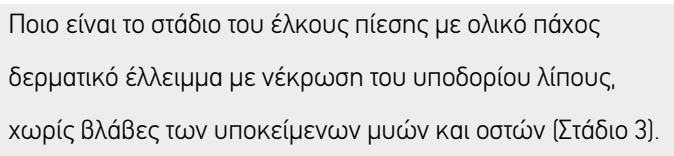 & $69(90,8 \%)$ & $10(83,3 \%)$ & 0,002 \\
\hline 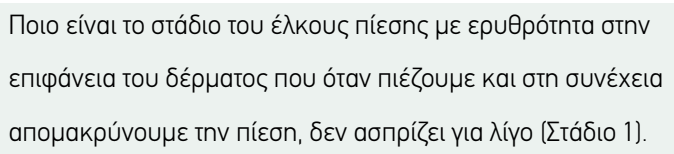 & $75(98,7 \%)$ & $10(83,3 \%)$ & 0,006 \\
\hline 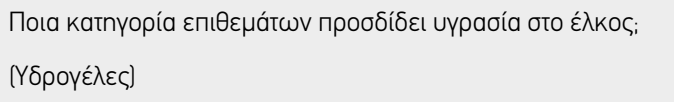 & $68(87,2 \%)$ & $8(72,7 \%)$ & 0,032 \\
\hline Aпаvтńбદıৎ & $18,7 \pm 2,8$ & $17 \pm 2,6$ & 0,036 \\
\hline 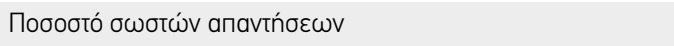 & $69,2 \pm 10,2$ & $62,9 \pm 9,7$ & 0,036 \\
\hline
\end{tabular}




\title{
Nursing personnel's knowledge regarding pressure sore management in a public hospital's medical and surgical wards
}

\author{
Pavlos Dimou', Michael Kourakos², Nikoleta Margari³, Evridiki Kamba3 ${ }^{3}$ Georgia Fasoi ${ }^{4}$, Martha Kelesi ${ }^{4}$ \\ 1. Nurse, MSc, Nikea's General Hospital "St Panteleimon" \\ 2. Nurse, MSc, PhD, Director of Nursing, General Hospital "Asklepeion" Voulas \\ 3. Assistant Professor, Faculty of Nursing, University of West Attica \\ 4. Professor, Faculty of Nursing, University of West Attica
}

\begin{abstract}
Introduction: Despite the recent advances in ulcer treatment and management, and the immense advancements in technology, pressure ulcers are still a major challenge for nurses and doctors who are responsible for their management.

Aim: The aim of the present study was to investigate the knowledge, practices and attitudes of nursing staff in a large public hospital regarding prevention and management of pressure ulcers.

Methods: This study is a research cross-sectional study which involved all nursing staff (nurses and nursing assistants) working in medical and surgical wards of the General Hospital of Nikaia "St Panteleimon". The questionnaire used for data collection has been developed by Gouda et al. (2014). The reliability of the questionnaire was high (Cronbach $a=0.714$ ) and the response rate was $76.7 \%$. Statistical analysis was performed with the SPSS for Windows (21.00) statistical software.

Results: The sample of the study consisted of 92 nurses aged $40.5( \pm 7.9)$ years. A total of 41 ( $44.6 \%)$ participants were nurse assistants, $33(35.9 \%)$ had graduate from applied science universities, $14(15.2 \%)$ had postgraduate degree and 4 ( $4.3 \%$ ) had a university degree. The participants' working experience was $15.1( \pm 9.1)$ years, while $45(48.9 \%)$ nurses were working in surgical departments and $47(51.1 \%)$ in medical ones. Of the total sample, twelve (13.3\%) nurses have claimed that training seminars on prevention and management of pressure ulcers have been organized in the hospital that they are working. In a total of 27 questions, the correct answers given by the respondents were $18.59( \pm 2.8)$ or $68.6 \%( \pm 10.4 \%)$ with a minimum of $12(44.4 \%)$ and a maximum of 25 $(92.6 \%)$ correct answers. Participants with a higher level of education and those who had attended educational seminars $[18.7( \pm 2.8)$ versus $17( \pm 2.6)]$ answered significantly more questions than those with a lower level of education $(p<0.05)$.

Conclusions: The nursing staff working in the medical and surgical wards had moderate levels of knowledge and high level of knowledge for the definition and classification of pressure ulcers. It is proposed that clinical protocols be drawn up concerning the staging, prevention and management of pressure ulcers, as well as a more organized continuing training of nursing staff.
\end{abstract}

Keywords: Knowledge, nursing staff, pressure ulcers, prevention, staging, treatment. 